\title{
The impact of diagnostic methods on the diagnosis of Clostridiodes difficile infection
}

\author{
E Nomlomo, MB ChB, DTM\&H, FC Path (SA) Clin; T Nana, MB BCh, DTM\&H, FC Path (SA) Micro \\ Microbiology Laboratory, Charlotte Maxeke Johannesburg Academic Hospital; and Department of Clinical Microbiology and Infectious Diseases, \\ National Health Laboratory Service and Faculty of Health Sciences, University of the Witwatersrand, Johannesburg, South Africa
}

Corresponding author: E Nomlomo (enomlomo@gmail.com)

\begin{abstract}
Background. Clostridiodes difficile is a common cause of healthcare-associated diarrhoea. Laboratory testing for C. difficile infection (CDI) remains an area of confusion, as there is not a single accepted reference standard or a single best test.

Objectives. To analyse the impact of different diagnostic methods on reported CDI rates. In addition, CDI incidence rates at Charlotte Maxeke Johannesburg Academic Hospital (CMJAH), Johannesburg, South Africa, were determined.

Methods. Results of stool samples submitted for C. difficile testing at CMJAH from 1 January 2014 to 31 August 2017 were reviewed. From January 2014 to July 2016, samples were tested by polymerase chain reaction (PCR) or toxin immunoassay, and from August 2016 to August 2017, algorithm-based testing (glutamate dehydrogenase and toxin immunoassay followed by PCR) was performed.

Results. A total of 4829 samples were submitted. For the first period, toxin immunoassay and PCR showed a positivity rate of $11.4 \%$ and $21.1 \%$, respectively, with an overall positivity rate of $18.7 \%$ (95\% confidence interval (CI) $15.6-21.9$ ). For the second period, the positivity rate was $15.9 \%$ (95\% CI 11.3 - 17.7). This rate included samples that were GDH-positive and either showed toxin production or had a positive Xpert result. The CDI incidence for the two periods was different, with an incidence rate of 8.8 and 6.1 per 10000 patient-days for the first and second periods, respectively.

Conclusions. The choice of laboratory testing method has a major impact on the diagnosis of CDI, and therefore on reported rates of CDI. Standardisation of laboratory testing and incidence rate reporting is required in order to obtain robust and reliable data.
\end{abstract}

S Afr Med J 2020;110(2):135-139. https://doi.org/10.7196/SAMJ.2020.v110i2.13684

Clostridiodes difficile is a common cause of healthcare-associated diarrhoea. The clinical outcome depends on host factors and the virulence of the toxin-producing strain. This organism causes disease that ranges in severity from asymptomatic colonisation to severe diarrhoea, pseudomembranous colitis, toxic megacolon, colonic perforation and death. ${ }^{[1-3]}$ The principal risk factor for C. difficile infection (CDI) is prior antibiotic therapy. ${ }^{[4]}$

Results of a systematic review assessing the epidemiology of CDI in low and middle Human Development Index countries show that there is a paucity of published literature describing the epidemiology and burden of CDI..$^{[2]}$ Rajabally et al.$^{[1,3]}$ highlight that data relating to the burden of C. difficile-associated disease (CDAD) in southern Africa are limited. The study conducted by this group emphasises that the magnitude of the CDI burden in South African (SA) hospitals is not known. ${ }^{[1,3]}$

Freeman et al ${ }^{[5]}$ note the two key issues that hinder understanding of the epidemiology of CDI. Firstly, there is poor recognition and documentation of CDAD ${ }^{[5]}$ The second obstacle is the many different approaches to the laboratory diagnosis of CDI. This is a dilemma also encountered in SA. ${ }^{[5]}$ There are numerous targets and combinations of targets that can be used to detect $C$. difficile (cell cytotoxicity assays (CCAs), toxigenic culture, glutamate dehydrogenase (GDH), toxins through enzyme immunoassay (EIA), and toxin genes through polymerase chain reaction (PCR)). Researchers in the UK have noted a variation in the reference standards for CDI diagnosis. This variation gives rise to differences in assessment of the diagnostic performance of assays and contributes to uncertainty in diagnosing CDI. ${ }^{[6,7]}$ It is therefore likely that the measured incidence of infection will vary according to the laboratory diagnostic method used..$^{[-7]}$
PCR-based testing is unable to differentiate between infection and colonisation. As a result, reliance on it as a stand-alone test may result in over-diagnosis of CDI. ${ }^{[3]}$ The low sensitivity of toxin EIAs also makes these unsuitable as stand-alone tests. ${ }^{[3,6]}$ For this reason, an algorithm that includes a sensitive assay (GDH or PCR) followed by a specific assay (toxin detection) may provide more accurate results. ${ }^{[8]}$ The Society for Healthcare Epidemiology of America (SHEA), the Infectious Diseases Society of America (IDSA) and the European Society of Clinical Microbiology and Infectious Diseases (ESCMID) all recommend the use of multistep algorithms for the diagnosis of CDI ${ }^{[8,9]}$ These algorithms allow reliable exclusion of CDI without additional tests if the GDH screen or PCR is negative, ${ }^{[8]}$ reducing the turnaround time significantly while providing accurate results. ${ }^{[8,9]}$

\section{Objectives}

Prior to July 2016, the Charlotte Maxeke Johannesburg Academic Hospital (CMJAH) Microbiology Laboratory used stand-alone PCR and stand-alone toxin EIA for CDI diagnosis. Currently, a two-step algorithm for CDI diagnosis is used. A rapid EIA for GDH and toxin $\mathrm{A} / \mathrm{B}$ is the initial test, followed by PCR on the subset of samples that are GDH-positive and toxin-negative. The primary objective of this study was to analyse the results yielded by the diagnostic methods used during the two periods and their impact on the reported rates of CDI. The secondary objective was to estimate the incidence of $\mathrm{CDI}$ in the overall patient population at CMJAH, as well as specific at-risk patient populations such as critical care, oncology, surgical and gastroenterology patients. 


\section{Methods \\ Design and setting}

This was a retrospective descriptive study. CMJAH is a 1088 -bed tertiary hospital in Johannesburg. Results of stool samples submitted for C. difficile testing at CMJAH from 1 January 2014 to 31 August 2017 were reviewed. Laboratory data were requested from the National Health Laboratory Service (NHLS) and Corporate Data Warehouse.

\section{Microbiological testing}

Formed stools were rejected by the laboratory. Samples with delayed transport to the laboratory ( $>2$ hours post collection) were not rejected. During the period January 2014 - July 2016, laboratory testing for CDI was performed using two test methods. The test method used in each case was determined by when the sample was submitted. Samples that were collected during the regular working hours of 07 h00 - 17h00 were analysed using PCR (Xpert C. difficile; Cepheid, USA). The test method used after hours, from 17h00 07 h00 and during weekends, was the EIA Immuno-Card Toxin A \& B assay (Meridian Bioscience, USA).

Samples that tested either PCR-positive with Xpert C. difficile or toxin-positive with the Immuno-Card Toxin A \& B assay were interpreted as positive for the purposes of analysis.

From 1 August 2016, a two-step algorithm was used for laboratory diagnosis of CDI. The C. Diff Quik Chek Complete (TechLab, USA) detects GDH as a screen for the presence of $C$. difficile. This assay also detects the presence of toxin $\mathrm{A}$ and $\mathrm{B}$. A positive result for both the $\mathrm{GDH}$ and toxin indicates $C$. difficile disease. In contrast, a positive result for only the GDH component may indicate the presence of a non-toxin-producing strain. For samples that tested GDH-positive and toxin-negative, reflex PCR (Xpert $C$. difficile; Cepheid) testing was performed to detect the presence of toxin-encoding gene.

Data for epidemiology review were sorted according to the current reporting and interpretation standard operation procedure at the NHLS (unpublished data): (i) GDH-negative and toxin-negative: C. difficile not detected; (ii) GDH-positive and toxin-positive: toxinproducing C. difficile detected; (iii) GDH-negative and toxin-positive: indeterminate results, repeat specimen to be submitted to the laboratory; and (iv) GDH-positive and toxin-negative: C. difficile antigen detected, toxin not detected. For the subset of samples that tested GDH-positive and toxin-negative following reflex PCR testing, data were also classified according to whether toxin-encoding genes were detected or not detected.

Samples that tested GDH-positive and toxin-positive were referred to as 'true positives' for the purposes of analysis. True positives together with samples that tested GDH-positive, toxin-negative and PCR-positive were referred to as 'all positives'. Samples with indeterminate results were excluded from the analysis.

Repeat samples within a 2 -week period were excluded from the analysis.

Patient-days data for calculation of incidence rates were obtained from the hospital. The combined PCR-positive and EIA-positive results were used for calculation of the incidence rates for the January 2014 - July 2016 period and 'all positives' for August 2016 - August 2017.

\section{Statistical analysis}

The positivity rate for each diagnostic method was calculated by dividing the positive CDI results by the total number of samples over the relevant periods (January 2014 - July 2016 and August 2016 August 2017).

The paediatric and adult patient-days for the relevant period were used as the denominator for the CDI incidence rate calculations.

Ninety-five percent confidence intervals (CIs) were determined for the incidence rates for the two periods.

Stata statistical software version 14 (StataCorp, USA) was used for the analysis.

\section{Ethical considerations}

The study was approved by the University of the Witwatersrand Human Research Ethics Committee (ref. no. M170941).

\section{Results}

A total of 650 samples tested positive from the 3463 samples collected from January 2014 to July 2016 (EIA and PCR methods). The overall positivity rate was $18.8 \%$ (95\% CI 15.6 - 21.9) (Table 1).

A total of 1366 samples were submitted during the second test period and 217 were positive, with a positivity rate of $15.9 \%$ (95\% CI 11.3 - 17.7). This rate included all the samples that were GDHpositive and either showed toxin production (54\%) or had a positive Xpert result (46\%) (Table 1).

The incidence density of $C$. difficile infections was higher in the first test period (Table 2).

For the study period August 2016 - August 2017, the multidisciplinary intensive care unit (ICU) had the highest incidence of CDI at 46.1 per 10000 patient-days. The second-highest incidence rate was seen in the trauma ICU, followed by the infectious disease unit, with rates of 29.1 and 27 per 10000 patient-days, respectively (Fig. 1). For many of the units, a large proportion of the positive results were toxin-negative, Xpert-positive.

Paediatric medical and paediatric surgical units had an overall low incidence rate of 2.1 and 1 per 10000 patient-days (Fig. 1).

\begin{tabular}{llll} 
Table 1. Impact of different laboratory testing strategies & & \\
\hline & $\begin{array}{l}\text { Total positive } \\
\text { samples, } \boldsymbol{n}\end{array}$ & $\begin{array}{l}\text { Total samples, } \\
\boldsymbol{N}\end{array}$ & $\begin{array}{l}\text { Positivity rate, } \\
\mathbf{\%}(\mathbf{9 5 \%} \mathbf{C I})\end{array}$ \\
\hline $\begin{array}{l}\text { Testing period January 2014 - July 2016 } \\
\quad \text { Xpert }\end{array}$ & 554 & 2622 & $21.1(19.5-23.1)$ \\
$\quad$ Meridian Immuno-Card & 96 & 841 & $11.4(10.8-12.2)$ \\
$\quad$ Total & 650 & 3463 & $18.8(15.6-21.9)$ \\
Testing period August 2016 - August 2017 & & & \\
$\quad$ C. Diff Quik Chek Complete (GDH-positive, toxin-positive) & 118 & 1366 & $8.6(7.9-9.2)$ \\
$\quad$ GDH-positive, toxin-negative and Xpert-positive & 99 & 1366 & $7.3(6.9-7.9)$ \\
$\quad$ Total & 217 & 1366 & $15.9(11.3-17.7)$ \\
CI = confidence interval. & & &
\end{tabular}


Table 2. Incidence density of Clostridiodes difficile

\begin{tabular}{llll}
\hline Test period & Total positive samples, $\boldsymbol{n}$ & Total patient-days & Incidence rate per $\mathbf{1 0} 000$ patient-days (95\% CI) \\
\hline January 2014 - July 2016 & 650 & 735240 & $8.84(8.17-9.55)$ \\
August 2016 - August 2017 & 217 & 357871 & $6.06(5.28-6.92)$ \\
CI = confidence interval. & & &
\end{tabular}

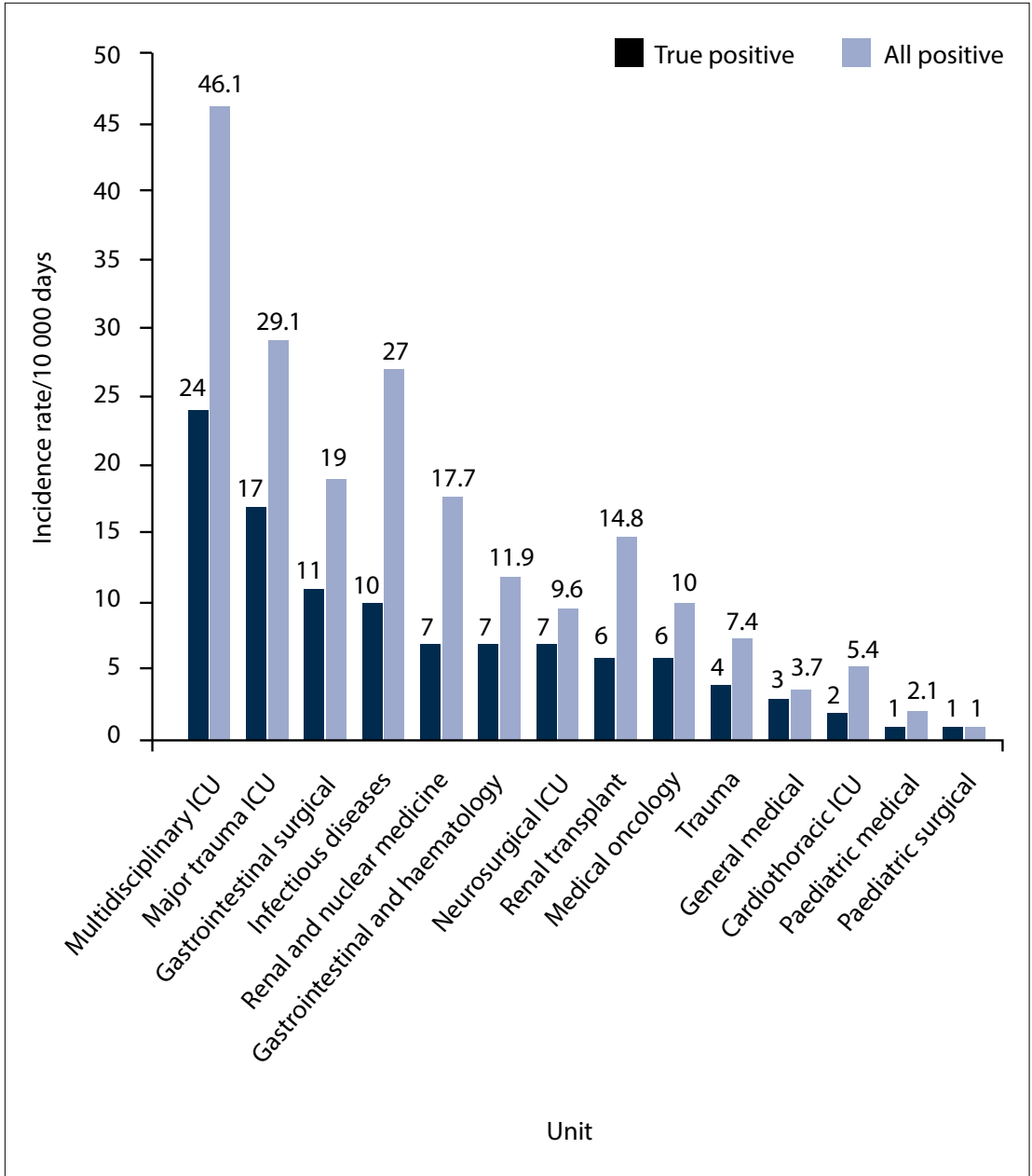

Fig. 1. Unit-specific Clostridiodes difficile incidence rate based on algorithm-based testing, August 2016 - August 2017. (ICU = intensive care unit.)

\section{Discussion}

The different laboratory testing strategies utilised by the CMJAH laboratory had a substantial impact on the reported CDI rates. In the first study period, stand-alone PCR-based testing yielded almost double the number of positive results compared with stand-alone toxin EIA testing. Similarly, in the second study period, reflex PCR-based testing of GDH-positive, toxin-negative samples almost doubled the number of positive results compared with GDH and toxin EIA testing alone. The difference in the laboratory testing methods and standalone PCR testing specifically, for a large proportion of the samples in the first study period, probably contributed considerably to the higher CDI incidence rate for the first study period. The overall CDI incidence rate at CMJAH is comparable to endemic rates reported from other regions (Table 2). ${ }^{[10]}$ Some hospital units had high CDI rates. However, a proportion of the positive results may reflect colonisation rather than CDAD.

Laboratory testing for CDI is a rapidly changing field. It is also an area of confusion, because there is not a single accepted reference standard or a single best test. ${ }^{[11]}$ The usefulness of a CDI diagnostic test is judged on its sensitivity, specificity, turnaround time, cost and availability. ${ }^{[1]}$ Toxigenic culture and CCA are regarded as the reference tests for the laboratory diagnosis of CDI. ${ }^{[6,8]}$ However, these assays are not widely used owing to their long turnaround times and technical requirements. ${ }^{[12]}$
These assays also pose a challenge in clinical interpretation and diagnostic method validations, as they have different targets and are not directly comparable..$^{[6,8]}$ Planche et al., ${ }^{[6]}$ in a study that compares results of toxigenic culture and CCA with clinical outcomes, reported that the two reference tests provide different data. This study shows that a positive CCA result correlates with $\mathrm{CDAD}$, whereas a positive toxigenic culture (with negative CCA) is more likely to indicate asymptomatic $C$. difficile colonisation. ${ }^{[6]}$ In keeping with these findings, the ESCMID review of CDI diagnostics concluded that when toxin is not detected from a sample but toxigenic culture is positive, further evaluation is required to distinguish asymptomatic colonisation from true CDI. ${ }^{[8]}$

During the first study period, PCR-based testing (Xpert) yielded a higher positivity rate than toxin EIA testing. PCR-based testing has been shown to be rapid, but is criticised for showing suboptimal specificity for CDI diagnosis. ${ }^{[11,12]}$ Increased CDI rates associated with PCR-based testing, compared with toxin detection-based diagnosis, have been reported in other studies. ${ }^{[6,12]}$ In a study assessing the impact of diagnostic assay type on CDI and the associated complication rates, Longtin et al. ${ }^{[12]}$ found that PCR alone was associated with a $>50 \%$ increase in CDI incidence compared with algorithm-based testing. ${ }^{[12]}$ PCR-based testing, like toxigenic culture, can have a low specificity, and hence a low positive predictive value (PPV), for CDI diagnosis and generally should not be used alone, but instead as part of a multistep algorithm. ${ }^{[6,8,9]}$ However, in high-prevalence settings or in patients with a high pre-test probability of CDI, PCR will have a more favourable PPV for CDI. ${ }^{[6,13]}$ The 2017 IDSA CDI guidelines support the use of standalone PCR in patients with a high pre-test probability of CDI. ${ }^{[9]}$

Studies have shown that toxin EIA tests are less sensitive than PCR and results are variable owing to toxin degradation associated with delayed sample testing. ${ }^{[3]}$ Delayed processing may have contributed to the lower positivity rate in the current study. In a study from the Western Cape, SA, researchers attributed a lower CDI burden to strain diversity and low levels of toxin 
production. ${ }^{[3]}$ In this study, the positivity rate of Xpert (21.13\%) was almost double that of EIA (11.41\%). Although toxin EIAs are rapid, widely available and inexpensive, they have poor sensitivity and are no longer recommended as stand-alone tests. ${ }^{[1]}$

In view of the increasing evidence that stand-alone assays for CDI diagnosis are suboptimal, the laboratory at CMJAH implemented a two-step algorithm in August 2016. Use of the two-step algorithm may have decreased the number of false-positive results and allowed for stratification of patients into those without CDI, those with CDI, and potential carriers for $C$. difficile. ${ }^{[6,8,9]}$

The majority of clinical laboratories do not use reference methods in multistep algorithms; ${ }^{[1]}$ however, the use of diagnostic methods that have been validated using the reference methods is common. ${ }^{[8]}$ Avila et al. ${ }^{[1]}$ showed that the combined sensitivity of assays such as C. Diff Quik Chek (GDH and toxin detection in a single assay) ranges between $80 \%$ and $100 \%$, and the specificity between $87 \%$ and $100 \% \cdot{ }^{[1]}$ However, in such assays the sensitivity of the toxin component is not certain, and there is therefore a role for PCR-based testing of GDH-positive, toxin-negative samples. ${ }^{[8,9]}$

The positivity rate of samples that were tested by a second test (Xpert) in the two-step algorithm was 7.25\%. The clinical relevance of samples that are GDH-positive, toxin-negative and PCR-positive is still not clear. The patient may have CDI with low toxin levels that are not detectable with the EIA used. Alternatively, the patient may simply be colonised with toxigenic C. difficile. ${ }^{[6,8]}$ The decision whether to treat these patients should be based on the pre-test probability of CDI and clinical evaluation of the patients. Colonised patients do, however, play a role in transmission of infection, and implementation of contact precautions is indicated. ${ }^{[8,9]}$

It is difficult to interpret the incidence data from the first study period, as the testing methodology used was not uniform. The majority of CDI cases were identified by PCR, and this may have resulted in a falsely elevated rate. Almost half the positive results in the second study period were toxin-negative, Xpert-positive; the true CDI incidence for this period may therefore be lower than the reported 6.1 cases per 10000 patient-days.

The incidence density of $C$. difficile was lower in the second study period compared with the first period. The testing rate in the two study periods differed (data not shown), and this may have had some impact on the incidence rates. However, CDI cases were defined using ESCMID- and IDSA-recommended algorithm-based testing during the second period, so the data are more robust than those of the first study period. ${ }^{[4]}$ The difference in incidence between the study periods is probably largely due to the move to a testing methodology (algorithm-based testing) that has been shown to have an increased accuracy for CDI diagnosis. ${ }^{[12,13]}$ Importantly, these results illustrate the need for uniform laboratory testing methodologies and surveillance definitions to promote reliable data generation, as well as improved interpretation and comparison of CDI incidence rates.

A CDI incidence rate of 5.3 per 10000 patient-days was found in a meta-analysis from Asia, which was similar to that reported in Europe and North America and indicated by the results of this study. ${ }^{[10]}$ Over the past few decades, there have been reports of widespread increases in the incidence and severity of and mortality associated with CDI. However, there is a paucity of SA data. Rajabally et al..$^{[1]}$ found that the incidence of CDI in their SA tertiary institution was much lower than that reported in the West. ${ }^{[1]}$ However, limitations of their study include that stand-alone toxin EIA testing was performed. The findings of the current study provide some of the much-needed local data. ${ }^{[1,2]}$
Analysis of the incidence density of specific units showed the multidisciplinary ICU to have the highest incidence rate. Critically ill patients are at increased risk for CDI owing to multifactorial causes. Risk factors include the use of broad-spectrum antimicrobials, gastric acid suppression, advanced age, prolonged hospitalisation, enteral feeds, chronic kidney disease and deficient immune status, all of which are associated with the development of $\mathrm{CDI} .^{[1,3,14-17]}$

Other units showing particularly high CDI incidence rates were the trauma, infectious diseases and gastrointestinal surgical units. Incidence rates were also elevated in the medical oncology, gastroenterology/haematology, renal transplant and renal/nuclear medicine units. Each of these patient populations have wellrecognised risk factors for CDI. ${ }^{[18,19]}$

Paediatric units showed an overall low incidence density. C. difficile is generally non-pathogenic in infants. ${ }^{[20]} \mathrm{CDI}$ may occur in children aged 1 - 3 years, but other aetiologies, especially viral, are more common. ${ }^{[20]}$ In older children, increasing CDI rates have been documented in some settings. ${ }^{[20,21]}$

This study has demonstrated that the overall reported CDI incidence rate decreased with the introduction of a two-step algorithm for laboratory diagnosis of CDI. Standardisation of laboratory testing methodology and CDI incidence rate reporting is required in order to yield robust data. Laboratories must incorporate detection of free toxin in their testing algorithms and clearly indicate on reports whether free toxin or toxigenic C. difficile has been detected. Clinicians must be cognisant of the difference in the implications of these two results.

\section{Study strengths and limitations}

Strengths of this study are that the results for a large number of samples were available for analysis, reducing potential bias towards any test method. During the first study period, all the samples were not tested by both PCR and toxin EIA; however, sample allocation to a particular method was based only on the time of sample receipt in the laboratory. In the second study period, the testing algorithm used was in alignment with international guidelines and recommendations. Similarly, the calculation of CDI incidence rates was based on recognised surveillance definitions.

The study is limited by its retrospective design, with potential to introduce bias. Our practice was restricted to the use of specific commercially available kits to perform EIA and PCR testing. The testing rate in the two study periods was not uniform, and definitive conclusions regarding the difference in the incidence rates therefore cannot be made. The clinical significance of GDH-positive, toxinnegative and PCR-positive results requires further elucidation. It is difficult to assess the magnitude of the overestimation of CDI rates related to PCR testing in both study periods. The study was conducted at a single centre, and caution should therefore be exercised in extrapolating these data to other settings.

\section{Conclusions}

C. difficile is a major cause of healthcare-associated diarrhoea. Laboratory diagnostics are important to differentiate CDI from other causes of diarrhoea and to establish the presence of infection v. colonisation. The choice of laboratory testing method(s) has a major impact on the diagnosis of CDI, and hence on the reported rates of CDI.

Declaration. This publication was a requirement for EN's MMed (Clinical Pathology) degree.

Acknowledgements. EN expresses her sincere appreciation to Dr Trusha Nana, clinical microbiology and infectious disease pathologist, for her 
unwavering support, encouragement and mentorship. It was a great honour to work under her supervision.

Author contributions. EM wrote the article. As her MMed supervisor, TN guided her with regard to the appropriateness of the content and directed her where there were gaps.

Funding. None.

Conflicts of interest. None.

1. Rajabally N, Pentecost M, Pretorius G, Whitelaw A, Mendelson M, Watermeyer G. The Clostridium difficile problem: A South African tertiary institution's prospective perspective. S Afr Med J 2013;103(3):168-172. https://doi.org/10.7196/SAMJ.6012

2. Forrester JD, Cai LZ, Mbanje C, Rinderkhencht TN, Wren SM. Clostridium difficile infection in lowand middle-human development index countries: A systematic review. Eur J Trop Med Int Health 2017;22(10):1223-1232. https://doi.org/10.1111/tmi.12937

3. Rajabally $\mathrm{N}$, Kullin B, Ebrahim K, et al. A comparison of Clostridium difficile diagnostic method for identification of local strains in a South African centre. J Microbiol 2016;65(4):320-327. https://doi. org $/ 10.1099 / \mathrm{jmm} .0 .000231$

4. Krutova M, Kinross P, Barbut F, Hajud A, Wilcox MH, Kuijper EJ. How to: Surveillance of Clostridium difficile infections. Clin Microbiol Infect 2018;24(5):469-475. https://doi.org/10.1016/j.cmi.2017.12.008 difficile infections. Clin Microbiol Infect 2018;24(5):469-475. https://doi.org/10.1016/j.cmi.2017.12.008
5. Freeman J, Bauer MP, Baines SD, et al. The changing epidemiology of Clostridium difficile infections. 5reeman J, Bauer MP, Baines SD, et al. The changing epidemiology of Clostridium
Clin Microbiol Rev 2010;23(3):529-549. https://doi.org/10.1128/CMR.00082-09

6. Planche TD, Davies KA, Coen PG, et al. Difference in outcome according to Clostridium difficile testing methods: A prospective multicenter diagnostic validation study of $C$. difficile infection. Lancet Infect Dis 2013;13(11):936-945. https://doi.org/10.1016/S1473-3099(13)70200-

7. Planche T, Aghaizu A, Holliman R, et al. Diagnosis of Clostridium difficile infection by toxin detection kits: A systematic review. Lancet Infect Dis 2008;8(12):777-784. https://doi.org/10.1016/S14733099(08)70233-0

8. Crobach MJT, Planche T, Eckert C, et al. European Society of Clinical Microbiology and Infectious Diseases: Update of the diagnostic guidance document for Clostridium difficile infection. Clin Microbiol Infect 2016;22(Suppl 4):S63-S81. https://doi.org/10.1016/j.cmi.2016.03.010
9. McDonald LC, Gerding DN, Johnson S, et al. Clinical Practice Guidelines for Clostridium difficile Infection in Adults and Children: 2017 Update by the Infectious Disease of America (IDSA) and Infection in Adults and Children: 2017 Update by the Infectious Disease of America (IDSA) and
Society for Healthcare Epidemiology of America (SHEA). Clin Infect Dis 2018;66(7):987-994. https:// Society for Healthcare Epide
doi.org/10.1093/cid/cix1085

10. Borren NZ, Ghadermarzi S, Hutfless S, Anantharishnan AN. The emergence of Clostridium difficile infection in Asia: A systematic review and meta-analysis of incidence and impact. PLoS One 2017;12(5):e0176794. https://doi.org/10.1371/journal.pone.0176797

1. Avila MB, Avila NP, Dupont AW. Recent advances in the diagnosis and treatment of Clostridium difficile infection. FI000Research 2016;5(16):1-5. https://doi.org/10.12688/f1000research.7109.1

12. Longtin Y, Trottier S, Brochu G, et al. Impact of the type of diagnostic assay on Clostridium difficile infection and complication rate in a mandatory reporting program. Clin Infect Dis 2013;59(1):67-73. https://doi.org/10.1093/cid/cis840

13. Yoldas O, Altindis M, Cufali D, Asik G, Kesli R. A diagnostic agorithm for the detection of Clostridium difficile-associated diarrhoea. Balkan Med J 2016;33(1):80-86. https://doi.org/10.5152/ balkanmedj.2015.15159

14. Deshpande A, Pasupuleti V, Rolston DDK, et al. Diagnostic accuracy of real-time polymerase chain reaction in detection of Clostridium difficile in stool samples of patients with suspected Clostridium difficile infection: A meta-analysis. Clin Infect Dis 2011;53(7):81-90. https://doi.org/10.1093/cid/cir50

15. O'Keefe SID. Tube feeding, the microbiota, and Clostridium difficile infection. World I Gastroentero 2010;16(2):139-142. https://doi.org/10.3748/wjg.v16.i2.139

16. Imlay H, Kaul D, Rao K. Risk factors for Clostridium difficile infection in HIV-infected patients. SAGE Open Med 2016; (4):1-5. https://doi.org/10.1177/2050312116684295

17. Longo DL. Clostridium difficile infection. N Engl J Med 2015;372(16):1539-1548. https://doi. org/10.1056/NEJMral403772

8. Evans CT, Safdar N. Current trends in epidemiology and outcomes of Clostridium difficile infection. Clin Infect Dis 2015;15;60(S2):S66-S71. https://doi.org/10.1093/cid/civ140

19. Alonso CD, Kamboj M. Clostridium difficile infection (CDI) in solid organ and hematopoietic stem cell transplant recipients. Curr Infect Dis 2014;16(8):414. https://doi.org/10.1007/s1 1908-014-0414-0

20. Lees EA, Miyajima F, Pirmohamed M, Carrol ED. The role of Clostridium difficile in the paediatric an neonatal gut - a narrative review. Eur J Clin Microbiol Infect Dis 2016;35(7):1047-1057. https://doi. org/10.1007/s10096-016-2639-3

21. Antonara S, Leber AL. Diagnosis of Clostridium difficile infection in children. J Clin Microbiol 2016;54(6):1425-1433. https://doi.org/10.1128/JCM.03014-15

Accepted 15 August 2019 